\title{
Efecto hipoglucemiante del extracto etanólico de Geranium ruizii Hieron. (pasuchaca) en la hiperglucemia inducida por aloxano en ratas
}

\author{
Hypoglycemic effect of Geranium ruizii Hieron. (pasuchaca) ethanolic \\ extract on alloxan-induced hyperglycemia in rats
}

\author{
Oscar Herrera-Calderon ${ }^{1,2}$, Rosa Chinchay-Salazar ${ }^{1}$, Estela Palomino-Ormeño1, \\ Evelyn Arango-Valencia ${ }^{1}$, Jorge Arroyo ${ }^{3,4}$ \\ 'Laboratorio de Fisiología y Fisiopatologia, Facultad de Farmacia y Bioquímica, Universidad Nacional San Luis Gonzaga, Ica, Perú. \\ ${ }^{2}$ Unidad de Posgrado, Facultad de Farmacia y Bioquímica, Universidad Nacional Mayor de San Marcos, Lima, Perú. \\ ${ }^{3}$ Laboratorio de Farmacología Experimental, Facultad de Medicina Humana, Universidad Nacional Mayor de San Marcos, Lima, Perú. \\ ${ }^{4}$ Instituto de Investigaciones Clínicas, Universidad Nacional Mayor de San Marcos, Lima, Perú.
}

\begin{abstract}
Resumen
Introducción: Geranium ruizii (Pasuchaca) es una planta medicinal utilizada tradicionalmente como hipoglucemiante en el departamento de Ancash, Perú. Objetivo: Evaluar el efecto hipoglucemiante del extracto etanólico de Geranium ruizii administrada en ratas con hiperglicemia inducida por aloxano. Diseño: Experimental. Institución: Laboratorio de Farmacología Experimental, Facultad de Medicina Humana, Universidad Nacional Mayor de San Marcos, Lima, Perú. Material biológico: Planta entera de Geranium ruizii, ratas Holtzman hembras de ocho semanas con $200 \pm 20 \mathrm{~g}$ de peso corporal. Intervenciones: La hiperglicemia fue inducida con aloxano. Las ratas incluidas en el estudio presentaron una glicemia $>200 \mathrm{mg} / \mathrm{dL}$. Se formaron seis grupos de seis ratas cada uno. El grupo I recibió agua destilada $2 \mathrm{~mL}$; Ios grupo II, III y IV recibieron Geranium ruizii $50 \mathrm{mg} / \mathrm{kg} ; 150 \mathrm{mg} / \mathrm{kg}$ y $300 \mathrm{mg} / \mathrm{kg}$, respectivamente (vía oral); al grupo V se administró glibenclamida $5 \mathrm{mg} / \mathrm{kg}$ y al grupo VI insulina $4 \mathrm{UI} / \mathrm{kg}$. Principales medidas de los resultados: Glucemia (mg/ $\mathrm{dL}$ ), porcentaje de inhibición del radical DPPH, especies reactivas al ácido tiobarbitúrico (TBARS) (nmoles/mL), estudio histológico de páncreas. Resultados: La dosis de $150 \mathrm{mg} / \mathrm{kg}$ de G. ruizii redujo 65,58\% los valores de glicemia a las $2 \mathrm{~h}$ post administración (Kruskal Wallis; $p<0,001$ ), redujo TBARS en $22,34 \%$ e inhibió el radical DPPH en 23,66\%; el tejido pancreático se mantuvo en buen estado de conservación. Conclusiones: El extracto etanólico de Geranium ruizii (pasuchaca) tuvo efecto hipoglicemiante en ratas con hiperglucemia inducida con aloxano.
\end{abstract}

Palabras clave: Geranium, hiperglucemia, efecto hipoglucemiante, TBARS, aloxano, antioxidante.

\section{Abstract}

Introduction: Geranium ruizii Hieron. (pasuchaca) is a medicinal plant used by traditional medicine to lower glycemia, in Ancash, Peru. Objective: To determine the hypoglycemic effect of Geranium ruizii ethanolic extract on alloxan-induced hyperglycemia in rats. Design: Experimental. Setting: Laboratorio de Farmacología Experimental, Facultad de Medicina Humana, Universidad San Marcos, Lima, Peru. Biological material: Geranium ruizii, eight weeks female Holtzman rats $200 \pm 20 \mathrm{~g}$ of body weight. Interventions: Experimental hyperglycemia (>200 mg/dL) was induced with alloxan. Rats were divided into six groups of six rats each. Group I received $2 \mathrm{~mL}$ distilled water, groups II, III, IV received respectively Geranium ruizii 50, 150 and $300 \mathrm{mg} / \mathrm{kg}$, group V glibenclamide $5 \mathrm{mg} / \mathrm{kg}$ and Group VI insulin 4IU/kg. Main outcome measures: Glycemic level (mg/dL), inhibition of DPPH (\%), reduction of reactive species to barbituric acid (TBARS) (nmoles/mL), histopathology study. Results: G. ruizii at $150 \mathrm{mg} / \mathrm{kg}$ reduced hyperglycemia $68.58 \%$ at $2 \mathrm{~h}$ following alloxan induction (Kruskal Wallis; $p<0.001$ ), reduced TBARS in vivo $22.34 \%$, and inhibited DPPH $23.66 \%$ to $1.0 \mathrm{ug} / \mathrm{mL}$; the pancreas tissue remained in good condition. Conclusion: The Geranium ruizii (pasuchaca) ethanolic extract showed hypoglycemic effect in alloxan-induced hyperglycemia in rats.

Keywords: Geranium, hyperglycemia, hypoglycemic effect, TBARS, alloxan, antioxidant.

\section{An Fac med. 2015;76(2):117-22 / dx.doi.org/10.15381/anales.v76i2.11135}




\section{INTRODUCCIÒN}

La Organización Mundial de la Salud (OMS) estima que más de 220 millones de la población mundial presentan diabetes y se proyecta que este número se duplicará para el año $2030^{(1,2)}$. La diabetes es una enfermedad cuyo marcador principal es la concentración elevada de glucosa debido a la disminución de insulina producida por las células $\beta$ del páncreas (diabetes tipo I) o a través de la pérdida de respuesta a la insulina por las células presentes en el tejido adiposo y muscular (diabetes tipo II) ${ }^{(3)}$.

El aloxano ha sido ampliamente usado para inducir diabetes mellitus experimentalmente, causando necrosis de las células $\beta$ del páncreas y originar la generación de radicales libres, con rol importante en la patogénesis de la diabetes mellitus del ser humano. Se ha demostrado que el aloxano induce la producción de peróxido de hidrógeno $\left(\mathrm{H}_{2} \mathrm{O}_{2}\right)$ y algunos radicales libres tales, como el oxígeno singlete $\left(\mathrm{O}_{2} \cdot\right)$ y el hidroxilo $\left(\mathrm{OH}^{-}\right)$, que produce daño seguido de la muerte celular ${ }^{(4)}$.

La familia Geraniaceae presenta aproximadamente 11 géneros, con casi 800 especies distribuidas en áreas templadas y subtropicales de ambos hemisferios ${ }^{(5)}$. Los principales metabolitos activos identificados en el género Geranium son los compuestos fenólicos tipo flavonoides glucosidados (quercetina, kempferol, rhamnetina) y ácidos fenólicos ${ }^{(6)}$. El Geranium ruizii Hieron. (pasuchaca) es una hierba perenne nativa del Perú perteneciente a la familia Geraniaceae, tiene el aspecto de un conjunto de pequeñas ramas que parten de un tallo bifurcado, restos de pecíolos y estípulas persisten en las ramas, las hojas son más o menos elípticas, de 5 por 6 a $7 \mathrm{~mm}$; florece en los meses de verano y se desarrolla en países como Perú y Bolivia ${ }^{(7,8)}$.

Estudios farmacológicos de algunas especies de Geranium han demostrado tener actividades biológicas como antidiabética, hipotensora, astringente, diurética, hepatoprotectora, antioxidante, antiinflamatoria y antiviral ${ }^{(9)}$.
Los flavonoides son un tipo de compuestos fenólicos naturales que se distribuyen en las plantas y estos compuestos son agentes potenciales antidiabéticos debido a que ejercen múltiples acciones como hipoglucemiante (acción insulinomimético) y antihiperglucémico (secretagogo de insulina) ${ }^{(10)}$. Se ha demostrado que el kaempferol-3, 7-O-(a)-dirhamnosido (kaempferitrina) y kaempferol-3-neohesperidosido actúan a través de múltiples mecanismos, constituyendo una fuerte evidencia de su rol insulinomimético en asegurar la homeostasis de la glucosa. La apigenina-6-C-(2"-O- $\alpha-\mathrm{L}-$ rhamnopiranosil)- $\beta$-L-fucopiranosido y apigenina- 6 -C- $\beta$ - $\mathrm{L}$-fucopiranosido actúan como secretagogos de insulina o como agentes insulinomiméticos ${ }^{(11)}$.

Los medicamentos sintéticos tienen muchos efectos adversos; por ello la búsqueda de nuevos antidiabéticos procedentes de fuentes naturales es una alternativa atractiva para el tratamiento de la diabetes mellitus, por su seguridad. La presente investigación tuvo por objetivo evaluar el efecto hipoglucemiante del extracto etanólico del Geranium ruizii Hieron. (pasuchaca) administrada en ratas con hiperglicemia inducida por aloxano.

\section{MÉTODOS}

La planta medicinal conocida como pasuchaca fue recolectada en el distrito de Tapacocha, provincia de Recuay departamento de Ancash, a $3394 \mathrm{msnm}$. Posteriormente fue identificada taxonómicamente en el Museo de Historia Natural de la Universidad Nacional Mayor de San Marcos (Constancia № 127-USM-2013). En la preparación del extracto etanólico, $2 \mathrm{~kg}$ de planta entera (raíz, tallo, hojas, flores) fue desecada bajo sombra durante un periodo de 2 semanas; se trituró manualmente y luego se maceró con $4 \mathrm{~L}$ de etanol al $96 \%$ por 7 días con agitación constante, protegido de la luz solar. Se filtró el macerado en papel Whatman № 1 para despues evaporar el solvente en una estufa a una temperatura no mayor de $40^{\circ} \mathrm{C}$, hasta obtener finalmente $43 \mathrm{~g}$ de extracto etanólico seco. Este proceso se desarrolló en el Laboratorio de Farmacología Experimental de la Facultad de Medicina de la Universidad Nacional Mayor de San Marcos.

Con relación al estudio fitoquímico preliminar según Lock, $1994^{(12)}$, cada reacción de identificación de metabolitos secundarios presentes en el extracto etanólico se realizó con $5 \mathrm{~mL}$ de extracto problema diluido y 5 gotas de reactivos de identificación para cada metabolito.

Para el modelo experimental, se utilizó ratas albinas cepa Holtzman, hembras, procedentes del Instituto $\mathrm{Na}$ cional de Salud. Las ratas fueron instaladas en el Bioterio de la Facultad de Medicina Humana de la UNMSM, en donde fueron aclimatadas durante 15 días a una temperatura entre $22-26^{\circ} \mathrm{C}$, con una humedad entre $60-75 \%$ y un ciclo de luz/oscuridad de 12/12 horas. Se les mantuvo con libre acceso al agua y alimentos. Se determinó el efecto hipoglucemiante en ratas, según Kameswara Rao y col., $1999^{(13)}$, con modificación en la dosis de monohidrato de aloxano a una dosis de $100 \mathrm{mg} / \mathrm{kg}$ disuelto en buffer citrato $0,3 \mathrm{M} \mathrm{pH} \mathrm{4,5}$ como inductor de hiperglicemia, por vía intraperitoneal a una sola dosis. Se determinó la glucosa basal al inicio del experimento y después de 48 horas de haber administrado el aloxano. Se incluyó en el estudio a los animales con glicemia mayor a $200 \mathrm{mg} / \mathrm{dL}$, seleccionando 36 ratas.

Se formó aleatoriamente seis grupos de seis animales cada uno, según el siguiente diseño experimental. Todas las sustancias fueron administradas por vía oral a excepción de la insulina, que fue por vía intraperitoneal. Grupo I: agua destilada $2 \mathrm{~mL}$ (control); Grupo II: G. ruizii 50 mg/kg; Grupo III: G. ruizii 150 $\mathrm{mg} / \mathrm{kg}$; Grupo IV: G. ruizii $300 \mathrm{mg} / \mathrm{kg}$; Grupo V: glibenclamida $5 \mathrm{mg} / \mathrm{kg}$; Grupo VI: insulina 4 UI/kg. Se administró los extractos una vez al día durante cuatro días consecutivos, a las 9:30 am. El dosaje de glicemia se realizó a las 2 
horas después de administrar el extracto, y luego cada 24 horas.

Para la medición del efecto hipoglucemiante, las muestras sanguíneas fueron colectadas del ápice de la cola del animal, desechando la primera gota y recibiendo la siguiente sobre la tira reactiva marca Medisense $(\mathbb{R})$ de Laboratorio Abbot y utilizando un glucómetro digital Optium $®$, siguiendo las instrucciones respectivas.

Al término del experimento, las ratas fueron anestesiadas por inhalación de éter y se colectó la sangre vía punción cardiaca para el análisis de especies reactivas al ácido tiobarbitúrico (TBARS) formado por la peroxidación lipídica. Se siguió la técnica de Buege y col., $1978^{(14)}$, expresando en $\mathrm{nmol} /$ $\mathrm{mL}$ de plasma que, al reaccionar con el ácido tiobarbitúrico (TBA), forma un complejo coloreado que fue leído a $535 \mathrm{~nm}$ en espectrofotómetro UV-VIS (UNICO-2100).

La actividad antioxidante in vitro se realizó mediante el método de neutralización del radical 1,1-difenil-2-picrilhidrazilo (DPPH) ${ }^{(15)}$, para lo cual se preparó solución metanólica de DPPH 0,01 mM, solución metanólica de Geranium ruizii $0,1,0,5$ y $1,0 \mu \mathrm{g} / \mathrm{mL}$ y patrón de referencia (trolox y vitamina C). Las muestras y los controles reaccionaron con el reactivo DPPH, realizándose la lectura a $517 \mathrm{~nm}$ luego de 30 minutos de reacción.

Para la evaluación histopatológica según Devi y col., $1998^{(16)}$, los páncreas

Tabla 1. Prueba preliminar cualitativa de los metabolitos secundarios del extracto etanólico de Geranium ruizii Hieron.

\begin{tabular}{ccc} 
Prueba de caracterización & Resultado & Metabolito secundario \\
Reacción de la ninhidrina & $(+)$ & Grupos aminos libres \\
Reacción de la gelatina & $(+)$ & Taninos \\
Reacción con cloruro férrico (FeCl3) & $(+)$ & Compuestos fenólicos \\
Reacción de Dragendörff & $(+)$ & Alcaloides \\
Reacción de Haeger & $(+)$ & Alcaloides \\
Reacción Mayer & $(+)$ & Alcaloides \\
Reacción de Shinoda & $(+)$ & Flavonoides \\
Reacción de Borntrager & $(+)$ & Quinonas \\
Reacción de Lieberman-Burchard & $(+)$ & Triterpeno y/o esteroides \\
Prueba de la espuma & $(+)$ & Saponinas \\
\hline
\end{tabular}

$(+)=$ Presencia.

fueron extirpados y lavados suavemente con solución salina, para remover la sangre y los detritos adheridos al tejido. Se les fijó en solución de formol al 0,1 M tamponado a $\mathrm{pH} 7$, por 7 días. El procesamiento de los tacos de parafina se realizó empleando un dispensador de parafina. Los tacos obtenidos fueron llevados a congelamiento a $<0^{\circ} \mathrm{C}$, por un lapso de 3 horas, para luego proceder al corte de $3 \mu$ de espesor con ayuda de un micrótomo. Después, se retiró la parafina en una estufa, para proceder a la coloración con hematoxilina y eosina.

La descripción de variables se realizó con medias, desviación estándar, porcentaje de reducción. Para la comparación estadística entre grupos se realizó la prueba de Kruskal-Wallis; y para la comparación de las medianas de los diferentes grupos tratados con el grupo control se utilizó la prueba de U de Mann Whitney. Se consideró significativo un valor $\mathrm{p}<0,05$, con intervalo de confianza del 95\%.

\section{RESULTADOS}

Al realizar el análisis fitoquímico, se identificó la presencia de numerosos metabolitos secundarios, como flavonoides, compuestos fenólicos, taninos, y alcaloides, observando cambios de color y precipitación (tabla 1).

Las ratas seleccionadas tuvieron un peso de $182,20 \pm 11,5 \mathrm{~g}$ y sus valores de glicemia antes de la inducción de la hiperglucemia experimental fueron de $85 \pm 4 \mathrm{mg} / \mathrm{dL}$. Con la inducción del aloxano se logró valores hiperglicémi-

Tabla 2. Efecto del extracto etanólico de Geranium ruizii (pasuchaca) sobre la hiperglicemia inducida por aloxano (A) en ratas.

\begin{tabular}{|c|c|c|c|c|c|c|}
\hline \multirow{2}{*}{$\begin{array}{l}\text { Grupo experimental } \\
\qquad(\mathrm{N}=6)\end{array}$} & \multicolumn{5}{|c|}{ Niveles de glucemia $(\mathrm{mg} / 100 \mathrm{~mL}){ }^{*}$} & \multirow{2}{*}{$\begin{array}{c}\text { Porcentaje de } \\
\text { efecto }(\%)\end{array}$} \\
\hline & Basal ** & $2 \mathrm{~h} * * *$ & $48 \mathrm{~h} * * *$ & $72 \mathrm{~h} * * *$ & $96 \mathrm{~h} * * *$ & \\
\hline I Control: Agua destilada $2 \mathrm{~mL}$ & $202 \pm 10,6$ & $183 \pm 13,9$ & $276 \pm 20,8$ & $305 \pm 10,8$ & $401 \pm 16,8$ & - \\
\hline II: Extracto $50 \mathrm{mg} / \mathrm{kg}$ & $315 \pm 18,2$ & $120 \pm 7,2^{a}$ & $131 \pm 6,9^{a}$ & $126 \pm 6,9$ a & $114 \pm 9,7^{a}$ & 60,13 \\
\hline III: Extracto $150 \mathrm{mg} / \mathrm{kg}$ & $305 \pm 17,1$ & $114 \pm 10,4^{\mathrm{a}}$ & $100 \pm 5,5^{a}$ & $96 \pm 5,5^{a}$ & $93 \pm 8,4^{\text {a }}$ & 65,58 \\
\hline IV: Extracto $300 \mathrm{mg} / \mathrm{kg}$ & $230 \pm 9,1$ & $156 \pm 6,18^{a}$ & $143 \pm 6,4^{\text {a }}$ & $123 \pm 6,4^{\text {a }}$ & $117 \pm 11,1^{\mathrm{a}}$ & 60,86 \\
\hline V: Glibenclamida $5 \mathrm{mg} / \mathrm{kg}$ & $286 \pm 15,5$ & $87 \pm 16,28^{b}$ & $95 \pm 5,8^{b}$ & $82 \pm 3,4^{b}$ & $87 \pm 5,9$ b & 70,90 \\
\hline VI: Insulina 4 UI/kg & $271 \pm 18,26$ & $84 \pm 8,26^{b}$ & $81 \pm 1,5^{b}$ & $81 \pm 1,5^{b}$ & $81 \pm 2,7 b$ & 72,96 \\
\hline
\end{tabular}

*Valores expresados en Media \pm Desviación estándar. ${ }^{* *}$ KrusKal Wallis $(p=0,09)$.

*** Variaciones de los niveles de glucemia post administración del extracto, Kruskal Wallis $(p<0,001)$. U de Mann Whitney entre el grupo control y los grupos con tratamiento a $(p<0,01), \quad b(p<0,001)$. 


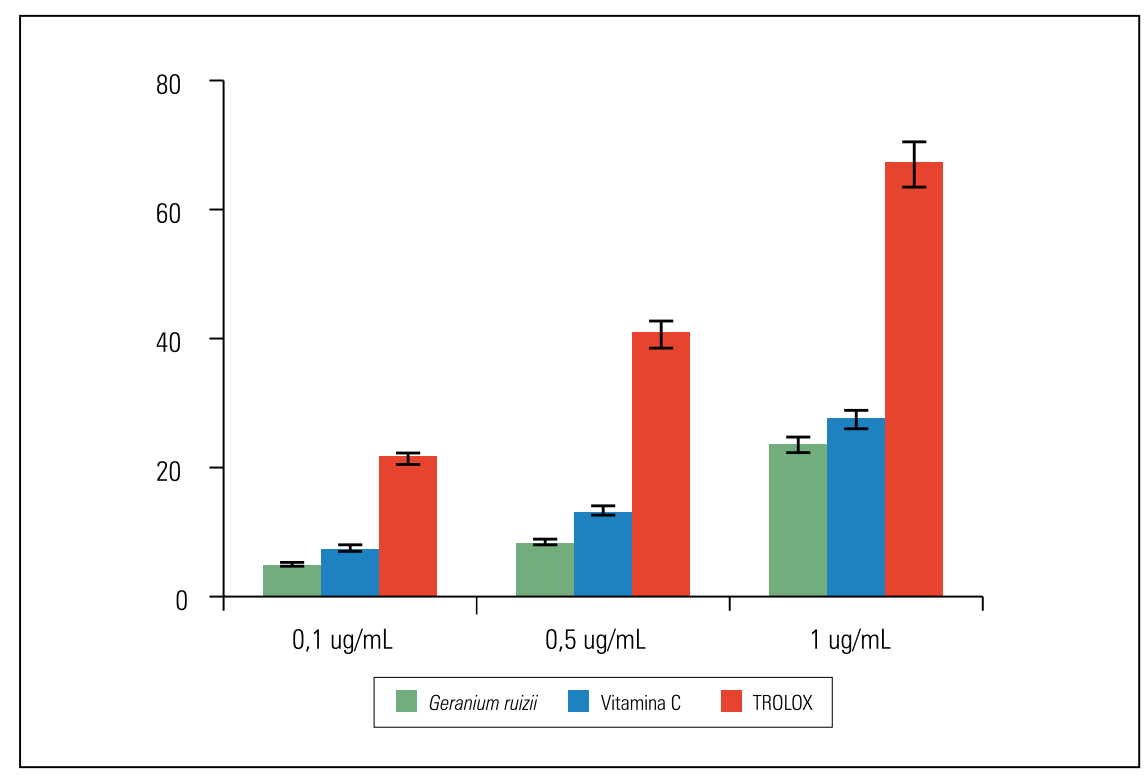

Figura 1. Actividad antioxidante in vitro del extracto etanólico de Geranium ruizii Hieron.

cos por encima de los $200 \mathrm{mg} / \mathrm{dL}$. Ver las medias y sus desviaciones estándar de las glicemias por grupo y horas en la tabla 2. El análisis de diferencia de medianas Kruskal Wallis reveló diferencia significativa $(p<0,01)$ entre los tratamientos. El grupo II (Geranium ruizii 50 $\mathrm{mg} / \mathrm{kg}$ ) y III (Geranium ruizii $150 \mathrm{mg} /$ $\mathrm{kg}$ ) presentaron una mediana menor de glicemia comparado con el grupo control (grupo I) que solo recibió agua destilada ( $p<0,01$, prueba de $U$ de Mann Whitney), en todas las horas evaluadas post administración del extracto. En el grupo III (Geranium ruizii $150 \mathrm{mg} / \mathrm{kg}$ ) la glicemia disminuyó significativamente $(p<0,01$, prueba de U de Mann Whit- ney) solo a las 2 horas y hasta el final de experimento.

Los niveles de TBARS también evidenciaron una reducción a la dosis de $150 \mathrm{mg} / \mathrm{kg}$ del extracto etanólico comparado con el grupo I ( $p=0,008$; U de Mann Whitney) (tabla 3). Los fármacos utilizados redujeron la glucosa sanguínea, pero sin efecto antioxidante in vivo en comparación al extracto etanólico de G. ruizii. En la evaluación de captación del radical DPPH, el porcentaje de actividad antioxidante fue $23,7 \%$ a la concentración de 1,0 ug/ $\mathrm{mL}$, menor en comparación al trolox y vitamina C (figura 1).

Tabla 3. Niveles de especies reactivas al ácido tiobarbitúrico (TBARS) expresados en nmoles $/ \mathrm{mL}$ de plasma.

\begin{tabular}{cccc} 
Grupo experimental* & TBARS & Porcentaje de reducción & ${ }^{*} \mathrm{p}$ \\
I: Agua destilada $2 \mathrm{~mL}$ & $0,94 \pm 0,35$ & - & - \\
II: Geranium ruizii $50 \mathrm{mg} / \mathrm{kg}$ & $0,78 \pm 0,21$ & 17,0 & 0,007 \\
III: Geranium ruizii $150 \mathrm{mg} / \mathrm{kg}$ & $0,73 \pm 0,26$ & 22,3 & 0,008 \\
IV: Geranium ruizii $300 \mathrm{mg} / \mathrm{kg}$ & $0,81 \pm 0,18$ & 13,8 & 0,007 \\
V: Glibenclamida $5 \mathrm{mg} / \mathrm{kg}$ & $0,88 \pm 0,36$ & 6,4 & 0,063 \\
VI: Insulina 4UI/kg & $0,87 \pm 0,39$ & 7,4 & 0,023 \\
\hline
\end{tabular}

Los datos de ninguno de los extractos presentó distribución normal ( $p<0,05$, Shapiro Wilk). TBARS expresados en X: media; DE: desviación estándar.

*p: comparación entre las medianas de las dosis del extracto y del aloxano $100 \mathrm{mg} / \mathrm{kg},(p<0,05$, Prueba de Mann-Whitney).

En el estudio anatomopatológico (figura 2) se observó una protección del páncreas frente a la exposición del tóxico inductor de hiperglicemia (aloxano), en las ratas tratadas con el extracto etanólico de G. ruizii, en los tres niveles de dosis, comparado con la glibenclamida e insulina, donde los islotes de Langerhans se mostraron esclerosados y en proceso de lisis celular.

\section{DISCUSIÓN}

En el presente estudio se evidenció que el extracto etanólico de Geranium ruizii a la dosis de 50 y $150 \mathrm{mg} / \mathrm{kg}$ (grupo II y III, respectivamente) tuvo efecto hipoglucemiante en ratas hembras albinas cepa Holtzman con hiperglicemia, cuando fue comparado con el grupo I (control). Ambos grupos empezaron su acción a partir de las 2 horas, y la respuesta hipoglucemiante fue sostenida hasta las 96 horas. Conforme pasó el tiempo, fue mayor la disminución de la glicemia, debido a la exposición continua del extracto que fue administrado hasta el último día del experimento. Todos los grupos tratados lograron una disminución significativa de la glicemia durante todas las horas de evaluación, con respecto al grupo I control. Los medicamentos como insulina y glibenclamida, reconocidos como hipoglucemiantes, demostraron su efecto de manera continua y sostenida.

Actualmente, los polifenoles como la curcumina, capsaicina, gingerol, catequinas, resveratrol y quercetina han demostrado sus efectos antiinflamatorios directamente bloqueando la ruta proteína quinasa activada por mitógeno (MAPK), actividad NFkB y la expresión de citoquinas antiinflamatorias. Se ha sugerido los efectos antinflamatorios que involucran al menos en parte la activación directa del receptor activador proliferador de peroxisomas $\gamma$ $(\operatorname{PPAR} \gamma)^{(20)}$. Este receptor es el regulador en varios aspectos del metabolismo de lípidos y diferenciación de adipocitos ${ }^{(21)}$. Otro importante mecanismo de los polifenoles es a través de las proteínas conocidas como sirtuinas. La sir- 


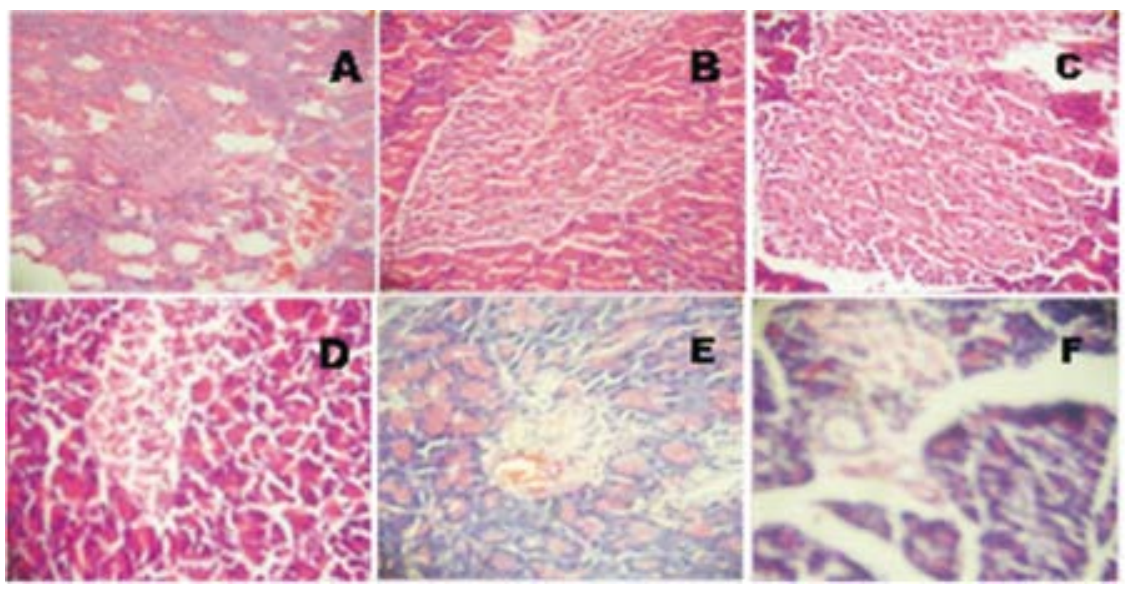

Figura 2. Microforografias de páncreas (islotes de Langerhans) de ratas tratadas con el extracto etanólico de G. ruizii en un modelo de hiperglicemia inducida por aloxano. 400 X. A: Grupo tratado con aloxano; se observa islote de Langerhans esclerosado y gran congestión intersticial. B: Grupo tratado con G. ruizii 50 mg/kg; páncreas normal. C: Grupo tratado con G. ruizii 150 mg/ kg, páncreas normal. D: Grupo tratado con G. ruizii 300 mg/kg, páncreas normal. E: Grupo tratado con glibenclamida $5 \mathrm{mg} / \mathrm{kg}$, se observa páncreas muy pequeño esclerosado. F: Grupo tratado con insulina $4 \mathrm{UI} / \mathrm{kg}$; se observa islotes de Langerhans lisándose.

los grupos experimentales que recibieron el extracto a los tres niveles de dosis evidenciaron protección (figura 2). Los animales que recibieron glibenclamida e insulina presentaron en alguna forma lesión pancreática, evidenciándose lisis y esclerosis a nivel de los islotes de Langerhans. Considerando que la obesidad ligada a la diabetes tipo II está asociada con un grado bajo de inflamación, se ha propuesto que los polifenoles pueden proteger de los daños producidos por la diabetes tipo II a través de sus efectos antiinflamatorios ${ }^{(28)}$.

Se concluye que el extracto etanólico de Geranium ruizii Hieron. (pasuchaca) a la dosis de $150 \mathrm{mg} / \mathrm{kg}$ presentó efecto hipoglucemiante en la hiperglicemia inducida por aloxano en ratas, además de brindar efecto protector al páncreas y efecto antioxidante in vivo e in vitro.

\section{AGRADECIMIENTOS}

deacetilasa tipo III implicado en el rol antienvejecimiento y antinflamatorio, que evidencia un rol protector en la enfermedad crónica de la diabetes, y la restricción calórica que produce al activarse directamente este receptor ${ }^{(22)}$. Recientemente, una SIRT1 dependiente de la deacetilación del PPAR $\gamma$ ha demostrado una forma de modulación selectiva para PPAR $\gamma$, permitiendo la inducción de los genes BAT y supresión de los genes WAT viscerales, asociados con la resistencia a insulina ${ }^{(23)}$. Por ello, los polifenoles modularían los mecanismos dependientes de AMPK-SIRT1PPAR $\gamma$, con rol importante como antinflamatorio, antiobesidad, antiesteatosis y efecto hipoglucémico, efectos de la acción sinérgica de varios metabolitos tipo polifenoles ${ }^{(24)}$. Por otro lado, es ampliamente aceptado que la diabetes mellitus incrementa la producción de radicales libres derivados del oxígeno, anión superóxido $(\mathrm{O} 2-)$, peróxido de hidrógeno ( $\mathrm{H} 2 \mathrm{O} 2)$ y radical hidroxilo $(\mathrm{OH}-)$, en cantidades superiores a los niveles fisiológicos, los que sobrepasan la capacidad antioxidante celular endógena ${ }^{(25)}$. En su mecanismo de detoxificación, el aloxano puede sobrepasar las barreras antioxidantes y formar especies reactivas que tienen como su principal blanco los lípidos de las membranas celulares ${ }^{(26)}$. Una manera de evaluar este daño es mediante la prueba de lipoperoxidación. En el ensayo in vivo, la dosis de $150 \mathrm{mg} / \mathrm{kg}$ redujo el contenido de TBARS $(0,73 \pm 0,26$ nmoles $/ \mathrm{mL}$; $\mathrm{p}<0,001)$. En el ensayo de capacidad antioxidante in vitro comparado con el trolox y vitamina $\mathrm{C}$, el extracto etanólico inhibió en 36\% el radical DPPH, lo que se correlaciona a lo observado en el modelo de medición in vivo. Esto se debería a que el extracto, al contener compuestos fenólicos de naturaleza flavonoidal, reduce la peroxidación lipídica al neutralizar la reacción en cadena de especies reactivas oxigenadas, puesto que los polifenoles son reconocidos por su propiedad de barredores de radicales libres por su propiedad de donar electrones. Su actividad antioxidante depende de la estabilidad de diferentes sistemas, así como también del número y la localización de grupos hidróxilos (27). En la presente investigación se ha demostrado que el aloxano produce lesión y muerte de las células $\beta$ del páncreas en animales de experimentación sin recibir tratamiento, mientras que
Al Dr. José Ernesto Ráez Gonzales, médico patólogo, profesor principal de la Facultad de Medicina Humana de la Universidad Nacional Mayor de San Marcos, por su aporte y colaboración en el presente estudio.

\section{REFERENCIAS BIBLIOGRÁFICAS}

1. Setacci C, de Donato G, Setacci F, Chisci E. Diabetic patients: epidemiology and global impact. J. Cardiovasc. Surg. 2009;50:263-73.

2. World Health Organization. Prevalence data of diabetes worldwide, 2009.

3. Schwarz PE, Li J, Lindstrom J, Tuomilehto J. Tools for predicting the risk of type 2 diabetes in daily practice. Horm Metab Res. 2009;41:86-9. doi: 10.1055/s-0028-1087203.

4. Abdul-Hamid M, Moustafa N. Protective effect of curcumin on histopathology and ultrastructure of pancreas in the alloxan treated rats for induction of diabetes. The Journal of Basic \& Applied Zoology. 2013;66;169-79.

5. Camacho-Luis A, Gayosso-De-Lucio J, Torres-Valencia Muñoz-Sánchez JL, Alarcón-Hernández E, López R, et al. Antioxidant constituents of Geranium bellum rose. J Mex Chem Soc. 2008;52(2):103-7.

6. Miliauskas G, Van Beek T, Venskutonis P, Linssen J, De Waard P, Antioxidative activity of Geranium macrorrhizum. Eur Food Res Technol. 2004;218:253-61.

7. Bussmann R, Paniagua-Zambrana N, Rivas M, Molina N, Cuadros M, Olivera J. Peril in the market-classification and dosage of species used 
as anti-diabetics in Lima, Peru. J Ethnobiol Ethnomed. 2013;9:37.

8. Geraniaceae, Geranium ruizii Hieron. Bot Jahrb Syst. 20(3, Beibl. 49): 31. 1895 [9 Apr 1895] Id 109277-2 Version: 1.3 View Record history.

9. Bussmann RW, Malca G, Glenn A, Sharon D, Nilsen $B$, Parris $B$, et al. Toxicity of medicinal plants used in traditional medicine in Northern Peru. J Ethnopharmacol. 2011;137(1):121-40. doi: 10.1016/j. jep.2011.04.071.

10. De Sousa E, Zanatta L, Seifriz I, Creczynski-Pasa TB, Pizzolatti MG, Szpoganicz B, et al. Hypoglycemic effect and antioxidant potential of kaempferol3,7-O-(alpha)-dirhamnoside from Bauhinia forficata leaves. J Nat Prod. 2004;67:829-32.

11. Cazarolli LH, Folador P, Pizzolatti M, Silva F. Signaling pathways of kaempferol-3-neohesperidoside in glycogen synthesis in rat soleus muscle. Biochimie. 2009;91:843-9.

12. Lock O. Investigación Fitoquímica. Métodos en e Estudio de Productos Naturales. 2da edición. Lima: Fondo Editorial Pontificia Universidad Católica de Perú; 1994:33-5.

13. Kameswara Rao B, Kesavulu M, Giri R, Apparao Ch. Antidiabetic and hypolipidemic effect of Momardica cymbalaria Hook fruit powder in aloxan diabetic rats. J Ethnopharm. 1996;67:103-7.

14. Buege J, Aust S. Microsomal lipid peroxidation. Methods in Enzymology. 1978;52:302-10.

15. Brand-Williams W, Cuvelier ME, Berset C. Use of a free radical method to evaluate antioxidant activity. Food Science and Technology. 1995;28(1):25-30.

16. Devi RS, Narayan S, Vani G, Shyamala Devi CS Gastroprotective effect of Terminalia arjuna bark on diclofenac sodium induced gastric ulcer. Chem Biol Interact. 2007;167(1):71-83

17. Elsner M, Gurgul-Conwey E, Lenzen S, Relative importance of cellular uptake and reactive oxygen species for the toxicity of aloxan and dialuric acid to insulin producing cells. J Biol Med. 2006:41:825-34

18. Nakamura U, Iwase M, Uchizono Y, Sonoki K, Sasaki N, Imoto H, et al. Rapid intracelular acidification and cell death by $\mathrm{H}_{2} \mathrm{O} 2$ and alloxan in pancreatic s cells. J Biol Med. 2006;40:2047-55.

19. Jakobs S, Fridrich D, Hofem S, Pahlke G, Eisenbrand G. Natural flavonoids are potent inhibitors of glycogen phosphorylase. Mol Nutr Food Res. 2005;50:52-7.

20. Floyd ZE, Wang ZQ, Kilroy G, Cefalu WT. Modulation of peroxisome proliferator-activated receptor $\mathrm{g}$ stability and transcriptional activity in adipocytes by resveratrol. Metabolism. 2008;57:S32-8.

21. Varga T, Czimmerer Z, Nagy L. PPARs are a unique set of fatty acid regulated transcription factors controlling both lipid metabolism and inflammation. Biochim Biophys Acta. 2011;1812:1007-22.

22. Penumetcha M, Santanam N. Nutraceuticals as ligands of PPAR $\gamma$. PPAR Res. 2012;2012:858352. doi.org/10.1155/2012/858352.

23. Prior RLE, Wilkes SR, Rogers T, Khanal RC, Wu $X$, Howard LR. Purified blueberry anthocyanins and blueberry juice alter development of obesity in mice fed an obesogenic high-fat diet. J Agric Food Chem. 2010;58:3970-6.

24. Beher D, Wu J, Cumine S, Kim KW, Lu SC, Atangan L, et al. Resveratrol is not a direct activator of SIRT1 enzyme activity. Chem Biol Drug Des. 2009;74:619-24. doi: 10.1111/j.1747-0285 2009.00901.x

25. Dal-Pan A, Blanc S, Aujard F. Resveratrol suppresses body mass gain in a seasonal non-human primate model of obesity. BMC Physiol. 2010;10:11. doi: 10.1186/1472-6793-10-11.

26. Qiang L, Wang L, Kon N, Zhao W, Lee S, Zhang $Y$, et al. Brown remodeling of white adipose tissue by SirT1-dependent deacetylation of PPAR gamma. Cell 2012;150:620-32. doi: 10.1016/j. cell.2012.06.027

27. Ghule A, Jadhav S, Bodhankar S. Effect of ethanolic extract of seeds of Linum usitatissimum (Linn.) in hyperglycaemia associated ROS production in PBMNCs and pancreatic tissue of alloxan induced diabetic rats. Asian Pacific J Trop Dis. 2012;2(5):405-10.

28. Simsek N, Kaya M, Kara A, Can I, Karadeniz A Kalkan Y. Effects of melatonin on islet neogenesis and beta cell apoptosis in streptozotocin induced diabetic rats: an immunohistochemical study. Domestic Anim. Endocrinol. 2012;43:47-57. doi: 10.1016/j.domaniend.2012.02.002

Artículo recibido el 21 de octubre de 2014 y aceptado para publicación el 26 de enero de 2015.

Conflictos de intereses.

Los autores expresan no tener algún conflicto de interés.

Correspondencia:

Mg. Oscar Herrera Calderon

Dirección: Departamento Académico de Ciencias Farmacéuticas, Universidad Nacional San Luis Gonzaga de Ica, Ciudad Universitaria, Panamericana Sur Km 305 s/n, lca Teléfono: (51)956550510

Correo electrónico: oh2000_4@hotmail.com oherreracalderon@gmail.com 